\title{
Iliopsoas Abscess in a 24-Months-Old Child: Management in Absence of an Identifiable Organism
}

\author{
Laraib Malik', Jawad Ahmed², Farheen Malik2*, Junaid Ahmad ${ }^{3}$ and Sina Aziz ${ }^{1}$ \\ 'Pediatrics Department, Abbasi Shaheed Hospital, Karachi, Pakistan \\ ${ }^{2}$ Department of Internal Medicine, Dow University of Health Sciences, Karachi, Pakistan \\ ${ }^{3}$ Liaquat University of Medical and Health Sciences, Jamshoro, Pakistan
}

\begin{abstract}
Iliopsoas abscess presents with vague and variable symptoms and is less commonly encountered in a clinical setting. The most common causative agent is staphylococcus aureus. In our case, a 2-year-old boy presented with high-grade fever, difficulty in walking, and pain in the right leg near the hip joint. Psoas sign was positive but his blood cultures were negative and CT scan showed a necrotic mass with peripheral enhancement measuring $7.0 \times 4.2 \times 2.6 \mathrm{~cm}$ in all three dimensions in right Psoas muscle. Biopsy of the abscess was denied and the patient was managed conservatively with intravenous ceftriaxone and metronidazole. This case highlights the importance of clinical examination and then co-relating it radiologically and focuses on the importance of broadspectrum antibiotics when necessary investigation (biopsy in our case) is denied.
\end{abstract}

Keywords: Pediatrics, iliopsoas abscess, unknown etiology, CT, ultrasound.

\section{INTRODUCTION}

Psoas muscle is located behind the peritoneum and originates from the lateral borders of last $\left(12^{\text {th }}\right)$ thoracic to 5 th lumber vertebra and inserts on the lesser trochanter of the femur. It is innervated by branches of $L 2, L 3$, and L4 from lumbar plexus, before the formation of the femoral nerve [1]. Psoas abscess was first described by Mynter in 1881 [2]. It is rare with an incidence of $0.4 / 100,000$ [3] and approximately 12 cases are reported worldwide per year [4].

A review of 124 cases published in 2009 yielded that Staphylococcus aureus was the most frequently identified organism in primary as well as abscess of skeletal origin. The most common organism isolated from the abscess of urinary and gastrointestinal tract origin was Escherichia coli [5]. Tuberculous abscess is most common in South Asia [6]. S. aureus is the most common organism in both developing and developed countries but Mycobacterium tuberculosis is uncommon in the western world. The incidence of iliopsoas abscess is higher in developing countries $[7,8]$. Most recent cases reported from Pakistan included a report on neonatal iliopsoas abscess [9] and a case of bilateral psoas abscess in an 18-year-old female [10]. In children, iliopsoas abscess is an unusual clinical finding with various etiologies and non-specific clinical presentation. This leads to delayed diagnosis and escalates morbidity and hospitalizations. A good clinical assessment, appropriate laboratory and radiological findings aid in making a precise diagnosis. We find it imperative to report this case to highlight the importance of clinical and radiological examination and the importance of broad-spectrum antibiotics when biopsy investigation is denied in case of a suspected psoas abscess. The timely empirical antibiotic treatment in such cases can improve the prognosis of patients.

\footnotetext{
*Corresponding Author: Farheen Malik, Department of Internal Medicine, Dow University of Health Sciences, Karachi, Pakistan;

Email:farheenmaalik@gmail.com

Received: October 24, 2019; Revised: January 09, 2020; Accepted: January 14, 2020

DOI: https://doi.org/10.37184/lnjpc.2707-3521.1.11
}

\section{CASE PRESENTATION}

A 2-year-old male child, completely vaccinated, weighing $14-\mathrm{Kg}$ presented to Abbasi Shaheed Pediatrics Department, Karachi on February 18, 2019, with complaints of high-grade fever for 15 days, difficulty in walking, and pain in the right leg near the hip joint for 2 days. The fever started gradually as low-grade over a period of 2 to 3 days and progressed to high-grade. It was continuous and not associated with rigor and chills. There was no history of trauma, tuberculosis contact, gastrointestinal or urinary complaints. Antenatal, natal and postnatal histories were non-significant.

The child appeared ill on general physical examination and he was lying with flexion of the hip joint on the right side. The patient was febrile (1010F) but vitally stable with no detectable lymphadenopathy. There were no signs of inflammation on local examination. The extension of the right hip joint was restricted and Psoas sign was positive. On examining the gait, he was limping with his right leg in mid-flexed position. The examination of rest of the systems was unremarkable.

Baseline investigations were performed. The patient was mildly anemic with hemoglobin $(\mathrm{Hb})$ of $10.7 \mathrm{gm} / \mathrm{dL}$,

Table 1: Lab values of the patient from admission to the last followup.

\begin{tabular}{l|c|c|c}
\hline \multicolumn{1}{c|}{ Parameters } & $\begin{array}{c}\text { At } \\
\text { Admission }\end{array}$ & $\begin{array}{c}\text { At } \\
\text { Discharge }\end{array}$ & $\begin{array}{c}\text { At Last } \\
\text { Follow-up }\end{array}$ \\
\hline $\mathrm{Hb}(\mathrm{gm} / \mathrm{dL})$ & 10.7 & 10.9 & 11.0 \\
\hline Total RBC $(\times 1012 / \mathrm{L})$ & 4.8 & 5.1 & 5.3 \\
\hline $\mathrm{MCV} \mathrm{(fL)}$ & 78 & 78 & 82 \\
\hline TLC $(\times 109 / \mathrm{L})$ & 18.4 & 9.3 & 5.8 \\
\hline Neutrophils $(\%)$ & 88.6 & 72.8 & 56.3 \\
\hline Platelet $(\times 109 / \mathrm{L})$ & 394 & 361 & 370 \\
\hline RFTs & Normal & Normal & - \\
\hline CRP $(\mathrm{mg} / \mathrm{dL})$ & 21 & 11 & 0.6 \\
\hline ESR $(\mathrm{mm} / \mathrm{hour})$ & 52 & 23 & 4 \\
\hline Size of Abscess on & $7.4 \times 1.8 \times 2.4$ & $2.8 \times 1.0 \times 1.1$ & $0.9 \times 0.6 \times 1.0$ \\
US & $\mathrm{cm}$ & $\mathrm{cm}$ & $\mathrm{cm}$ \\
\hline
\end{tabular}


leukocytosis $(18.4 \times 109 / \mathrm{L})$, and a normal platelet count $(394 \times 109 / L)$. Ongoing inflammation was confirmed by elevated Erythrocyte Sedimentation Rate (ESR) and C-reactive Protein (CRP) levels (52 mm/hour and $21 \mathrm{mg} /$ $\mathrm{dL}$ respectively). Renal functions were normal. Mountox test was negative and blood culture showed no growth. Lab reports of the patient are presented in Table 1. Findings on plain-film radiography of the right hip were unremarkable (Appendix Fig. 1). Ultrasound (US) hip

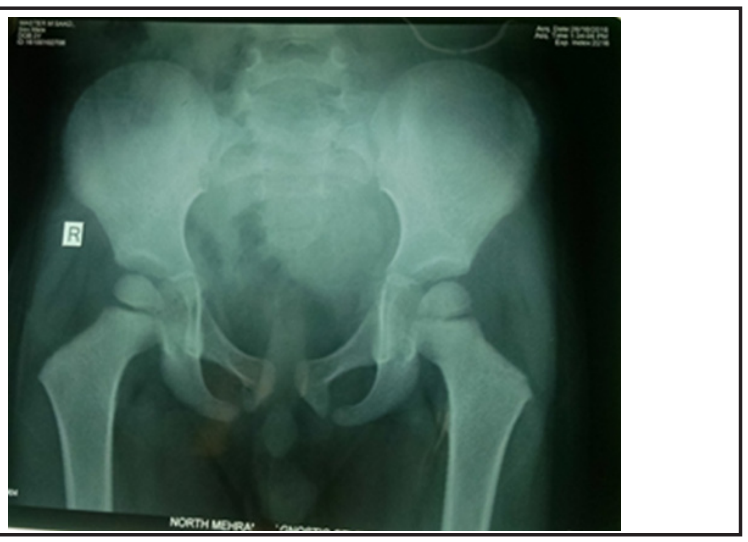

Fig. (1): X-ray pelvis showing normal findings on right side.

joint showed a hypoechoic collection in the right lower abdomen measuring $7.4 \times 1.8 \times 2.4 \mathrm{~cm}$ approximately. Computed Tomography (CT) scan of the whole abdomen with contrast (Appendix Fig. 2) showed a large necrotic soft tissue density mass measuring $7.0 \times 4.2 \times 2.6 \mathrm{~cm}$ in

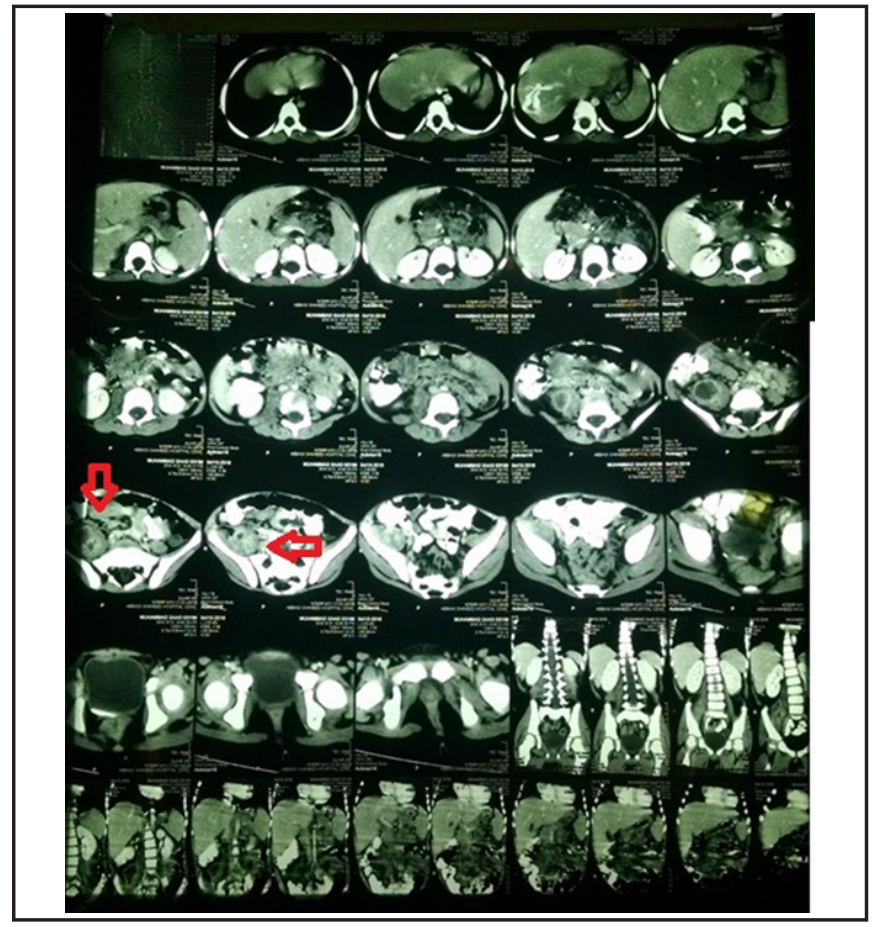

Fig. (2): CT abdomen showing iliopsoas abscess (red arrows).

all three dimensions in right Psoas muscle. In the postcontrast study, it showed peripheral enhancement. It was associated with fat stranding in surrounding fat. Superiorly the abscess extended up to the level of L2-3 vertebral disc. Inferiorly it extended down to the level of lesser trochanter of right femur.
The patient was started on broad-spectrum intravenous (IV) antibiotics (ceftriaxone $100 \mathrm{mg} / \mathrm{Kg} /$ day dose 6 hourly and metronidazole $30 \mathrm{mg} / \mathrm{Kg} / \mathrm{day}$ divided into 8-hour doses) that were continued for 4 weeks and then orally for 2 weeks. The patient's condition improved and fever started to subside after a week. After two weeks, his ultrasound showed a reduction in the size of the abscess (from $7.4 \times 1.8 \times 2.4 \mathrm{~cm}$ to $3.8 \times 1.4 \times 1.7 \mathrm{~cm}$ ) and the patient was discharged after 4 weeks. The patient was kept on close follow-up (every 2 weeks) for 1 month and he was afebrile without any difficulty in walking. His US showed further reduction in size at each follow-up until it disappeared by 3rd visit. The patient did not follow-up further despite the doctor's advice. Informed consent for publication of this case was obtained from the patient's parents.

\section{DISCUSSION}

Iliopsoas abscess has a variable clinical presentation. Patients may present with complaints of fever, pain in lower back or abdomen, limp, weight loss, and a lump in the groin region [7]. The classic triad of back pain, fever, and limp are found in less than one-third of cases of iliopsoas abscess [11]. The chief complaints in our case were fever, difficulty in walking and hip pain, and pain upon any attempt to move the leg at the hip joint. However, no mass or lump was seen in the groin or inguinal region. A precise diagnosis can be made by a good physical examination, laboratory, and radiological findings.

Patients of iliopsoas abscess find greatest comfort in lying in supine position with a moderately flexed knee and slightly externally rotated hip [12]. Iliopsoas abscess should be well-thought-out if the patient complains of pain on attempting flexion and external rotation of the hip with flexed knee. Iliopsoas inflammation causes contraction and pain in the iliopsoas muscle. This test can be useful in the diagnosis of iliopsoas abscess [13]. Although baseline laboratory investigations are non-specific, leukocytosis and elevated levels of CRP and ESR can be detected [1]. Our patient's labs were consistent having a high, ESR, CRP, and leukocytosis.

Iliopsoas abscess is more frequent in the younger population than older [7, 9]. The age of our patient was 2 years. The cause of a primary abscess is unclear. It can either be due to hematogenous spread from hidden infection or local trauma which may lead to hematoma formation within the muscle, this makes the psoas muscle prone to the formation of abscess [7]. Secondary iliopsoas abscess is mostly associated with gastrointestinal diseases such as Crohn's disease, appendicitis, and diverticulitis; also associated with urinary tract infections, vertebral osteomyelitis, septic arthritis, and sacroiliitis [7, 14]. Predisposing factors to primary iliopsoas abscess include diabetes mellitus, IV drug abuse, renal failure and immunosuppression such as from AIDS [7]. In our case, the patient had no predisposing factors, no underlying disease, and no history of any recent infection, gastrointestinal disease or trauma.

Breese et al. examined 142 pediatric cases diagnosed with iliopsoas abscess in Texas Children's Hospital, Houston and found that more than half $(57 \%)$ the 
abscesses were located on the right side, followed by $40 \%$ on the left side. Only $3 \%$ of cases had bilateral psoas abscess [15]. This is similar to our case, as our patient presented with a unilateral localization on the right side as well.

The abscess factor can lead to the presence of microorganisms in blood cultures. The commonly isolated organisms in primary and secondary abscess are S. aureus, followed by E. coli and Bacteroides spp. Other notable organisms that can be identified in blood culture include Mycobacterium tuberculosis $\left(4^{\text {th }}\right.$ most common organism identified), Streptococcus viridans, Enterococcus faecalis and Peptostreptococcus spp [5]. It is not uncommon to find Mycobacterium tuberculosis as a causative agent of iliopsoas abscess in developing countries [7]. Contrarily, in our case as the patient's blood culture showed no growth; while abscess culture could not be procured as the child was very irritable and parents did not give consent.

Ultrasonography (USG) is simple, reasonable and free from radiation; this method identifies $60 \%$ of the cases. A CT scan can provide better view of the retroperitoneal area, and the sensitivity of imaging modalities increase when both the US and CT are performed [16]. Our patient was also diagnosed through similar radiological modalities.

The treatment of psoas abscess involves drainage of the abscess and antibiotic therapy. Septic complications can occur if the abscess is not drained. Antibiotics are preferred, based on the culture of abscess material, but anti staphylococcal therapy should be started as soon as possible in patients suspected to have primary psoas abscess. Antibiotic therapy is only sufficient in cases of small abscesses with an early diagnosis. Patients that develop a neurologic deficit and show no response to medical treatment require surgical intervention [17]. This was contrary to our case as our patient's blood culture showed no growth; while abscess culture could not be procured. Our patient was started on empirical broad-spectrum antibiotics. Mortality rates are higher in secondary psoas abscess $(18.9 \%)$ than primary abscesses $(2.4 \%)$. In untreated cases, the prognosis can be very poor with a mortality rate of up to $100 \%$ [4]. In particular, age over 65 years, bacteremia and E. coli growth in culture media were found to be associated with mortality [5]. The risk of mortality in our patient was low as none of these factors were present in our case. The limitation to our case was the impossibility to obtain psoas biopsy for organism culture due to lack of consent therefore, no specific microbial etiology could be established for the abscess.

\section{CONCLUSION}

In conclusion, psoas abscess is rare and intricate to diagnose because of vague symptoms, which results in a delayed diagnosis, complications, and ultimately death if left untreated [18]. Therefore, iliopsoas abscess should be kept as a differential in patients presenting with difficulty walking and pain in the region of lower back, hip, groin, and leg. Diagnostic modalities, particularly USG and CT scan, should be used. In a scenario when the biopsy is denied and no identifiable organism can be obtained patient should be managed with broadspectrum antibiotics.

\section{ABBREVIATIONS}

C-reactive protein (CRP)

Computed tomography (CT)

Erythrocyte sedimentation rate (ESR)

Hemoglobin $(\mathrm{Hb})$

Intravenous (IV)

Ultrasonography (USG)

Ultrasound (US)

\section{CONFLICT OF INTEREST}

The authors declare no conflict of interest.

\section{ACKNOWLEDGEMENTS}

Laraib Malik and Jawad Ahmed contributed to study design and manuscript preparation. Farheen Malik and Junaid Ahmad contributed to manuscript preparation and critical review. Sina Aziz contributed to manuscript preparation. All authors have read and approved the manuscript.

\section{REFERENCES}

1. Taiwo B. Psoas abscess: a primer for the internist. South Med $\mathrm{J}$ 2001; 94(1): 2-5.

2. Mynter H. Acute psoitis. J Buffalo Med Surg 1881; 21: 202-10.

3. Bartolo D, Ebbs S, Cooper M. Psoas abscess in Bristol: a 10-year review. Int J Colorectal Dis 1987; 2(2): 72-6.

4. Gruenwald I, Abrahamson J, Cohen O. Psoas abscess: case report and review of the literature. J Urol 1992; 147(6): 1624-6.

5. López VN, Ramos JM, Meseguer V, Arellano JLP, Serrano R, Ordóñez MAG, et al. Microbiology and outcome of iliopsoas abscess in 124 patients. Medicine 2009; 88(2): 120-30.

6. Moore S, Rafii M. Imaging of musculoskeletal and spinal tuberculosis. Radiol Clin North Am 2001; 39(2): 329-42.

7. Mallick I, Thoufeeq M, Rajendran T. Iliopsoas abscesses. Postgrad Med J 2004; 80(946): 459-62.

8. Ricci MA, Rose FB, Meyer KK. Pyogenic psoas abscess: worldwide variations in etiology. World J Surg 1986; 10(5): 834-42.

9. Nisar MU, Sikander S, Noorain Z, Baig M-u-NS, Akhtar N. Primary iliopsoas abscess in a neonate. J Coll Physicians Surg Pak 2019; 29(6): S45-S7.

10. Shah HU, Khan MS, Zada N, Shah I, Shah AU, Mohammad R. Bilateral psoas abscess in out patients department district head quarter teaching hospital Bannu. EC Clin Exp Anat 2019; 3(1): 1-5.

11. Chern C-H, Hu S-C, Kao W-F, Tsai J, Yen D, Lee C-H. Psoas abscess: making an early diagnosis in the ED. Am J Emerg Med 1997; 15(1): 83-8.

12. Wong J. The psoas signs. Well-described but often forgotten physical examination findings of iliopsoas inflammation. N C Med J 1993; 54(11): 598-600.

13. Shields D, Robinson $P$, Crowley $T$. Iliopsoas abscess-a review and update on the literature. Int J Surg 2012; 10(9): 466-9.

14. Riyad MNY, Sallam MA, Nur A. Pyogenic psoas abscess: discussion of its epidemiology, etiology, bacteriology, diagnosis, treatment and prognosis-case report. Kuwait Med J 2003; 35: 44-7.

15. Bresee JS, Edwards MS. Psoas abscess in children. Pediatr Infect Dis J 1990; 9(3): 201-6.

16. Huang JJ, Ruaan MK, Lan RR, Wang M-C. Acute pyogenic iliopsoas abscess in Taiwan: clinical features, diagnosis, treatments and outcome. J Infect 2000; 40(3): 248-55.

17. Tomich EB, Della-Giustina D. Bilateral psoas abscess in the emergency department. West J Emerg Med 2009; 10(4): 288-91.

18. Kumar S. Psoas abscess: aetiological and clinical review. Rev Med Microbiol 2017; 28(1): 30-3. 